DEVOS, Hannes, RANCHET, Maud, BOLLINGER, Kathryn, CONN, Amber, AKINWUNTAN, Abiodun Emmanuel, 2018, Performance-based visual field testing for drivers with glaucoma: A pilot study, Traffic Injury Prevention, 19, 7, Taylor \& Francis Ltd, pp. 715-721, DOI: $10.1080 / 15389588.2018 .1508834$

\title{
Manuscript title: Performance-Based Visual Field Testing for Drivers with Glaucoma: A Pilot Study
}

Authors: Hannes Devos, $\mathrm{PhD}^{1}$, Maud Ranchet, $\mathrm{PhD}^{2}$, Kathryn Bollinger, $\mathrm{MD}^{3,4}$, Amber Conn, BS, OT ${ }^{1}$, Abiodun E. Akinwuntan, $\mathrm{PhD}, \mathrm{MPH}, \mathrm{MBA}^{5}$

\section{Affiliations:}

1. Department of Physical Therapy and Rehabilitation Science, School of Health Professions, The University of Kansas Medical Center, Kansas City, KS.

2. Univ Lyon, IFSTTAR, TS2, LESCOT, F-69675, LYON, France

3. Department of Ophthalmology, Medical College of Georgia, Augusta University, Augusta, GA.

4. Culver Vision Discovery Institute, Medical College of Georgia, Augusta University, Augusta, GA.

5. Dean's office, School of Health Professions, The University of Kansas Medical Center, Kansas City, KS, USA.

Address corresponding author: Hannes Devos, PhD, University of Kansas Medical Center, Department of Physical Therapy and Rehabilitation Science, MS 2002, Kansas City, KS 66160, hdevos@ kumc.edu, tel: 913-5882840, fax: 913-588-9135. 


\begin{abstract}
Objective: Conventional visual field (VF) tests are limited in predicting on-road driving performance of individuals with glaucoma. We developed a new performance-based VF test in a driving simulator for individuals with glaucoma. The aim was to evaluate the psychometric properties of the newly developed test.

Methods: In this pilot study, 17 drivers with glaucoma aged $65.24 \pm 9.69$ and 13 control drivers aged $61.27 \pm 11.45$ completed the performance-based VF task. Construct validity was determined by comparing performance on the new task with results on conventional visual and perimetry tests including Humphrey Visual Field Analyzer, Keystone Vision Screener, and Useful Field of View (UFOV®). Concurrent validity was evaluated by comparing performance on the new task with on-road driving performance, scored using the Test Ride for Investigating Practical fitness-todrive (TRIP). Ten individuals (seven with glaucoma and three controls) participated in the test-retest reliability assessment.

Results: Drivers with glaucoma identified fewer symbols ( $\mathrm{p}=0.047)$ and took longer to respond to the symbols $(\mathrm{p}=0.048)$ compared with controls. In the glaucoma group, correct responses on the performance-based VF test correlated strongly $(\mathrm{r}=-0.51, \mathrm{p}=0.046)$ with $\mathrm{UFOV} \circledast$ divided attention. Both glaucoma and control groups achieved submaximal to maximal scores on the TRIP (median (Q1-Q3) glaucoma: 193 (191-196); controls: 196 (195-196); $\mathrm{p}=0.16)$. No strong correlations were found between scores on the performance-based VF test and on-road driving performance in glaucoma. The intra-class correlation coefficients ranged between 0.77 for response time and 0.92 for correct responses, indicating good to excellent test-retest reliability.

Conclusions: We established the construct validity and test-retest reliability of the performance-based VF test. Future studies should include a larger sample with more severe driving difficulties to demonstrate the concurrent validity between performance-based VF testing and on-road driving performance in glaucoma.
\end{abstract}

Keywords: driving; glaucoma; driving simulator; visual field 


\section{INTRODUCTION}

Older drivers are the fastest growing segment of the population.(Dickerson et al. 2011) In the United States (US), the number of drivers older than 65 years of age has increased 33 percent from 2006 to 2015. (Loughran et al. 2007; National Highway Traffic Safety Administration 2017) As drivers age, their on-road driving safety may be impacted by age-related changes to eyesight. Open-angle glaucoma will affect over three million Americans by 2020.(Friedman et al. 2004) This disease is characterized by progressive damage to the optic nerve, resulting in irreversible loss of peripheral visual field (VF) and eventually blindness.(Jonas et al. 2017) The high prevalence of glaucoma amongst aging drivers calls for a performance-based VF test to assist health care professionals in determining fitness-todrive.(Medeiros et al. 2012)

Seemingly easy, driving is a highly automated, yet complex instrumental activity of daily living that requires intact visual, physical, sensory, and cognitive functions.(Ball et al. 2007) One of the basic requirements for safe driving is adequate VF.(Desapriya et al. 2014) Drivers with VF restrictions such as glaucoma are more prone to failing an onroad driving evaluation and incur more motor vehicle crashes (MVC) compared to older drivers with no VF loss.(Gramer and Gramer 2017; Harrabi et al. 2015; Haymes et al. 2007; Haymes et al. 2008; Kubler et al. 2015; Kunimatsu-Sanuki et al. 2016; Kwon et al. 2016; McGwin et al. 2005; Owsley et al. 1998; Szlyk et al. 2002; Szlyk et al. 2005; Wood et al. 2016)

Driving license authorities in many states of the US require minimum VF for driving.(Carr et al. 2010) The binocular VF standards extend in range from 105 to 150 degrees across the horizontal median.(Carr et al. 2010; Steinkuller 2010) Static VF tests such as the Keystone Vision Screener and the Humphrey Visual Field Analyzer are typically used to evaluate and predict the driving risks of drivers with glaucoma.(Gracitelli et al. 2015; Haymes et al. 2008; Kunimatsu-Sanuki et al. 2015; Kunimatsu-Sanuki et al. 2016; Tatham et al. 2015; Wood et al. 2016; Yuki et al. 2017)

However, the evidence for a direct link between VF loss, tested in static conditions, and impaired driving performance is at best inconclusive.(Wood and Black 2016) Driving requires accurate and timely detection of relevant stimuli in a constantly changing, cluttered environment. In such highly complex tasks, the area of visual field from which information can be extracted, tends to shrink, making stimuli in the periphery become more difficult to detect. (Ball et al. 1988) The ability to pay attention to the VF under situations of increased cognitive demand was initially described as "functional visual field", and later coined "useful field of view".(Edwards et al. 2006) This phenomenon becomes even more apparent in drivers with glaucoma.(Bentley et al. 2012; Gangeddula et al. 2017; Lee et al. 2017) In a driving simulator experiment, Gangeddula et al. examined the effect of increasing levels of cognitive demand on VF between drivers with and without glaucoma. When both optic flow and a psychomotor driving activity were added to the VF task, drivers with glaucoma showed disproportionately more shrinkage in VF compared to control drivers.(Gangeddula et al. 2017)

The Useful Field of View (UFOV®) test was developed to better capture functional VF by assessing speed of visual processing under increasingly complex task demands.(Ball et al. 1990) The UFOV® test is more predictive of driving 
safety than static visual field tests for drivers with glaucoma.(Gracitelli et al. 2015; Haymes et al. 2007) Yet, the UFOV® test only evaluates 10-30 degrees of horizontal field of view, depending on what version is used, and does not account for the visual flow and the psychomotor activity of steering and pedal operation that is typical of driving.(Gangeddula et al. 2017)

The limitations associated with static VF tests (Humphrey Visual Field Analyser, Keystone Vision Screener) and the currently available functional VF tests (UFOV®) prompted us to develop an ecologically valid, performance-based VF test in a setting that mimics real-life driving. Such simulated driving test could assist health care professionals in the decision making process of fitness-to-drive in individuals with glaucoma. In contrast to static VF tests, driving simulators can place the same demands on perceptual-motor processes and cognitive functions as real-life driving.(Medeiros et al. 2012; Wilkie and Mole 2017) Driving simulators are able to accurately reproduce static visual field tests in a contextual, standardized, safe and reliable setting.(Gangeddula et al. 2017; Wilkie and Mole 2017) Few studies have demonstrated the validity of simulator-based driving skills against performance on the road in glaucoma.(Tatham et al. 2015) The aims of this pilot study were (1) to compare performance on the newly developed performance-based VF test between drivers with glaucoma and controls, and (2) to investigate the psychometric properties (construct validity, concurrent validity, and test-retest reliability) of the new test.

\section{METHODS}

\section{Participants and Recruitment}

Thirty-three participants were recruited between February 20, 2015 and April 16, 2016 from the Department of Ophthalmology at Augusta University, Augusta, GA, or through word-of-mouth and flyer advertisement. The testing included two to three visits over the course of one week. Of the 20 individuals with glaucoma who consented, 17 completed the on-road test. Of the 13 controls who consented, 11 completed the on-road test. Ten participants (seven glaucoma patients and three controls) were invited back within two weeks to establish test-retest reliability.

Eligibility criteria for both groups included: (1) ophthalmologist diagnosis of open-angle glaucoma exemplified by optic nerve damage and VF loss (only for glaucoma patients); (2) valid driver's license; (3) self-reported mileage of at least 500 miles in the year prior to testing; and (4) devoid of other visual, neurological, internal or psychiatric conditions that may interfere with driving.

\section{Ethics}

Ethical approval was granted by the Augusta University's Institutional Review Board to conduct the study and consent was obtained from each participant.

\section{Questionnaires}

Demographic data such as age, sex, and years of education were collected. Participant's driving history was obtained via a standardized interview with questions pertaining to years of driving experience, annual mileage, and number of 
self-reported car crashes and traffic violations in the previous five years. The medical history included presence of comorbidities, prior eye surgeries and visual field impairments.

\section{Cognitive Battery}

The Montreal Cognitive Assessment (MoCA),(Nasreddine et al. 2005) Trail Making Test (TMT) A \& B,(Reitan 1986) and Stroke Driver Screen Assessment (SDSA)(Akinwuntan et al. 2013) were conducted to assess cognitive functions relevant to driving. The MoCA is a 30 point screening tool for different cognitive domains of language, orientation, attention, memory recall, and visuospatial/executive functions.(Nasreddine et al. 2005) The TMT A measures visuomotor speed by connecting 25 numbered circles in ascending order. The TMT B measures visuomotor speed and attentional shift by alternating between numbers and letters.(Reitan 1986) Time to complete TMT A and B were recorded. The four subtests of the SDSA (i.e., dot cancellation, directions, compass, and road sign recognition) jointly measure visual scanning, sustained attention, complex reasoning, divided attention, visual comprehension, and traffic knowledge.(Akinwuntan et al. 2013; Nouri and Lincoln 1993) Time and errors on the dot cancellation subtest, and scores on the directions, compass, and road sign recognition subtests were recorded.

\section{Performance-based Visual Field Test}

A desktop, low-fidelity driving simulator, which consisted of a computer with three 22 "' screens, providing a horizontal field of view of $100^{\circ}$ and a vertical field of view of $20^{\circ}$, was powered by STISIM Drive software (STI Inc., Hawthorne, CA). The simulator computer was connected to Logitech® steering wheel and pedals (Supplementary Figure 1).

After a familiarization period, participants completed the evaluation scenario, which lasted an average (standard deviation) of 17.78 (6.33) minutes, and depicted day-to-day driving events in city, residential, and rural traffic. Subjects were required to maintain their lane, change lanes, adapt their speed, negotiate curves, and make full stops at zebra crossings and traffic lights while obeying the traffic rules. In addition, unexpected and potentially hazardous events were simulated, such as a car suddenly pulling out from the shoulder of the road and a child crossing the street. Computer-generated measures of driving performance included: total crashes, speed exceedances, correct stops at traffic lights, center line crossings, and road edge excursions.

During the simulated drive, red squares (targets) were randomly displayed one by one throughout the VF provided by the three computer screens (Figure 1). Supplementary Figure 2 illustrates all 114 targets that were randomly projected horizontally and vertically in 5 degree increments and diagonally in 22.5 degree increments from the middle of the center screen ( 0 degrees). The target symbols dimensions $(2 \times 2 \mathrm{~cm})$, red color and configuration (Supplementary Figure 2) were based on other visual tests, and are described further in the study by Gangeddula et al. (2017). Subjects were instructed to press the button on the steering wheel as soon as the target was recognized on the screen. The VF outcome measures included number of correctly identified target symbols (accuracy), number of missed responses, and response time to the correctly identified VF symbols. Information about screen and room conditions is detailed in our previous work.(Gangeddula et al. 2017) No participants had to abort the driving 
simulation because of subjective or objective reports) of the simulator adaptation syndrome as measured with the Simulator Sickness Questionnaire.(Kennedy et al., 1993)

\section{Emergency Brake Test}

Participants were instructed to make five emergency stops (including one practice trial) in a 3-mile scenario by slamming on the brakes as soon as a red stop sign appeared in the center of the screen. The complex response time was composed of seeing time (time between stop sign display and releasing gas pedal); movement time (time between releasing gas pedal and pushing brake pedal), and brake reaction time (time between pushing brake pedal and coming to complete stop).

\section{Visual Battery}

VF was assessed using the Humphrey Visual Field Analyzer SITA Fast 24-2 (Humphrey Instrument, Dublin, USA) and the Keystone Vision Screener (Visionary Software version 2.0.14). Monocular VF outcomes from the Humphrey test included mean deviation (MD) and pattern standard deviation (PSD)(Chauhan et al. 2008) for better and worse eye. The outcome on the VF assessment from the Keystone Vision Screener included the degrees of binocular horizontal field of view and the pass-fail scores on a test of vertical field of view. Near, mid (26"), and far binocular visual acuity was also assessed on the Keystone with the denominator as outcome. For example, a subject that reported 20/40 binocular acuity scored 40 on the test. Lower scores indicated better visual acuity.

Functional VF was assessed with the UFOV® test, version 7.1.0 from Visual Awareness, Inc.(Ball et al. 1990) The UFOV ${ }^{\circledR}$ consisted of three parts: speed of processing, divided attention, and selective attention. Participant's performance was scored in milliseconds on a scale ranging between $17 \mathrm{~ms}$ and $500 \mathrm{~ms}$, with a lower score indicating better performance and greater functional visual field. In addition, a risk assessment (RA) score was calculated based on participant's overall performance on the three tasks. This 5-point ordinal RA score is used to indicate risk of prospective car crashes.

\section{On-Road Driving Test}

The on-road test was administered by a trained driving instructor registered with the Georgia Department of Driver Services who was blind to the medical condition of the participants. The on-road test was conducted in a Georgia Department of Driver Services approved vehicle with automatic transmission and adapted with dual controls for safety. The standardized route navigated urban, rural and interstate roadways within greater Augusta, GA. Each test took approximately 45 minutes to complete and was conducted during daylight hours. Evaluation of on-road driving performance was scored using the reliable and valid Test Ride for Investigating Practical fitness-to-drive (TRIP) checklist.(Akinwuntan et al. 2005) The total TRIP score is the sum of the scores of each of the 49 sub-items. These sub-items were scored on an ordinal scale ranging between 1=poor; $2=$ insufficient; $3=$ sufficient; and 4=good. Each participant was given a TRIP score immediately after completion of the road test, by the same trained assessor.

\section{Statistical Analysis}


Kolmogorov-Smirnov tests were used to determine normality of variables. Normally distributed interval/ratio variables were described using means (standard deviations), whereas not normally distributed interval/ratio and ordinal variables were descried using median $(\mathrm{Q} 1-\mathrm{Q} 3)$. Nominal variables were expressed as frequencies (\%). Between group differences in variables were compared using independent t-tests, Wilcoxon rank sum tests, or Fisher's Exact tests, as appropriate. Pearson $r$ or Spearman $\rho$ correlations were calculated to determine associations between the VF tests and on-road driving performance within the glaucoma and control groups (concurrent validity). The same statistics were used to investigate associations between the new VF test and other visual tests (construct validity). According to Cohen, values ranging between 0.10 and 0.29 reflect weak correlations, between 0.30 and 0.49 moderate correlations, and values of 0.50 and higher indicate strong correlations.(Cohen 1992) Intra-class correlations (ICC) were calculated to determine test-retest reliability of the performance-based VF measures. ICC values higher than 0.75 were considered excellent; values between 0.60 and 0.74 were considered good.(Cicchetti 1994) The consistency of measurements was verified graphically using the method of Bland and Altman (1986). $P$ values of less than 0.05 were considered significant. All analyses were conducted using SAS Enterprise Guide, version 6.1.

\section{RESULTS}

Overall, few statistically significant differences were observed between the glaucoma ( $\mathrm{n}=17$; age (SD) 65.24 (9.69); 9 men), and control groups ( $\mathrm{n}=11$; age (SD), 61.27 (11.45); 2 men) (Supplementary Table 1). Yet, the glaucoma group reported more prior eye surgeries and comorbidities compared with the control group. Nine individuals with glaucoma had undergone trabeculoplasty. Two controls reported pseudophakia. Hypertension was the highest reported comorbidity (ten participants with glaucoma and two controls). The two groups also performed equally well on all tests of the cognitive battery, except for the compass test, where drivers with glaucoma scored worse compared to the control group (15.65 (7.32) vs $23.82(8.18) ; \mathrm{p}=0.01)$.

\section{Between-group Comparisons of Simulator and Visual Performance}

The glaucoma group identified fewer VF symbols (98 (45 - 105) vs 107 (98 - 110); $\mathrm{p}=0.047)$ and took more time $(1.10 \mathrm{~s}(0.90-1.20)$ vs $0.90 \mathrm{~s}(0.80-1.05) ; \mathrm{p}=0.048)$ to detect the VF symbols compared with the control group (Table 1). No differences were found in any of the simulated driving performance measures, including brake response time, number of crashes, speed exceedances, and correct stops at stop signs.

We also compared performance on the standard visual and attention tests between drivers with glaucoma and controls (Supplementary Table 2). No differences were found between the two groups, except for worse eye MD of the Humphrey Visual Field Analyser (-5.74 (-2.03 - -10.57) vs $(-1.20(-0.45--2.80) ; \mathrm{p}=0.007)$ and the selective attention subtest of the UFOV® $(500(200-500)$ vs $219.95(123.50-273.30) ; \mathrm{p}=0.04)$.

\section{Concurrent Validity}

Drivers with glaucoma did not perform worse on the TRIP test compared to controls (193 (191 - 196) vs (196 (195 196); $\mathrm{p}=0.16$ ). In the glaucoma group, complex response time of the emergency stop task correlated strongly (Spearman 
rho=-0.57; $\mathrm{p}=0.04 ; \mathrm{n}=13$ ) with performance on the on-road TRIP test, indicating that longer response times were associated with poorer on-road driving performance (Supplementary Figure 3a). None of the performance-based VF tests in the driving simulator correlated with on-road driving performance. Participants with slower processing speed on the UFOV® performed more poorly on the road test (Spearman rho=-0.53; $\mathrm{p}=0.03 ; \mathrm{n}=17$; (Supplementary Figure 3b), although this correlation was heavily biased by an extreme value. The Spearman rho coefficient between processing speed and on-road driving performance was $-0.42(\mathrm{p}=0.11)$ when the extreme value was omitted from analysis $(\mathrm{n}=16)$. Participants with higher MD scores of the better eye performed better on the road test (Spearman rho=0.62; $\mathrm{p}=0.01 ; \mathrm{n}=$ 15; Supplementary Figure 3c; after removal of two extreme values, Spearman rho=0.42; $\mathrm{p}=0.13 ; \mathrm{n}=13$ ).

For controls, correct response time on the performance-based VF test was the only variable that correlated strongly with total TRIP scores (Spearman rho=-0.66; $\mathrm{p}=0.03 ; \mathrm{n}=11$; Supplementary Figure 3d). After omitting the extreme value, the correlation decreased to $-0.53(\mathrm{p}=0.11 ; \mathrm{n}=10)$. By contrast, none of the traditional VF tests significantly correlated with on-road driving performance.

\section{Construct Validity}

In the glaucoma group, participants who responded faster on the UFOV@ divided attention subtest, identified more symbols on the performance-based VF test (Spearman rho=-0.51; $\mathrm{p}=0.046 ; \mathrm{n}=16$; Supplementary Figure 3e).

Participants who identified more VF symbols on the performance based VF test were more likely to score better on total horizontal FOV of the Keystone vision screener (Spearman rho=0.59; $\mathrm{p}=0.02 ; \mathrm{n}=15$; Supplementary Figure 3f). For controls, response time of the performance-based VF test correlated significantly with total horizontal FOV of the Keystone vision screener (Spearman $\mathrm{rho}=-0.77, \mathrm{p}=0.01 ; \mathrm{n}=10$; Supplementary Figure $3 \mathrm{~g}$ ).

\section{Test-retest Reliability}

The ICC values of the performance-based VF test were $0.78(95 \%$ CI $0.09-0.95 ; p=0.02)$ for response time and $0.92(95 \%$ CI $0.68-0.98 ;$ p=0.0001) for correct responses. Figure 2 shows the Bland-Altman plots for test-retest reliability. All but one participant fell within 2 standard deviations of the mean difference of response time (Figure 2A) and mean difference of correct responses (Figure 2B). The mean difference of response times was 0.03 (95\% confidence interval (CI); -0.30 - 0.36). The Bland Altman plots showed less consistency for correct responses. The mean difference of correct responses was $-1.3(-8.69-6.09)$.

\section{DISCUSSION}

The purpose of this pilot study was to evaluate the psychometric properties of a newly developed performance-based VF test for drivers with glaucoma. We found preliminary evidence that the newly developed VF test (1) discriminates between drivers with glaucoma and controls; (2) correlates with other (functional) VF tests; and (3) shows good to excellent test-retest reliability. Yet, concurrent validity could not be determined in this pilot study.

The glaucoma group identified fewer symbols and spent more time locating the symbols while driving. These results align with the work by Gangeddula et al. that showed a shrinkage in functional VF in drivers with glaucoma.(Gangeddula et al. 2017) This finding is important given that greater VF deficits may increase the risk of 
MVC's in glaucoma.(Haymes et al. 2007; McGwin et al. 2005) In addition, drivers with glaucoma responded more slowly to the VF symbols, which may have implications on their ability to timely respond to potentially hazardous events in the periphery. Our result is in agreement with the impaired performance of drivers with glaucoma on peripheral detection tasks as observed in a previous simulator study.(Prado Vega et al. 2013) Likewise, on-road driving studies showed that drivers with glaucoma show deficiencies in detecting unexpected hazards along the road.(Haymes et al. 2008; Wood et al. 2016)

By contrast, only one variable of the static VF tests (MD worse eye of the Humphrey Analyzer) and one variable of the UFOV® test (selective attention) discriminated between patients and controls. The results of the Humphrey Analyzer showed that the glaucoma group had mild to moderate VF defects, which may explain why only few differences in static VF tests were found between patients and controls. The performance-based VF test may therefore be particularly indicated for mild to moderate glaucoma.

Despite poorer performance on the performance-based VF task, drivers with glaucoma did not show impairments in the simulated driving task. Although the driving simulator had a horizontal field of view of 100 degrees, the majority of the driving events, including observing road signs and speedometer, and responding to other traffic users, occurred in the central VF. This may explain why drivers with glaucoma did not perform worse on the driving tasks. Yet, the longer response times for the performance-based VF test suggest that drivers with glaucoma struggled attending to the driving task and to the peripheral symbols simultaneously. Similarly, Prado Vega et al found that drivers with glaucoma performed worse on a secondary visual task despite similar performance in primary driving tasks such as lateral vehicle control and obstacle avoidance.(Prado Vega et al. 2013) It is conceivable that drivers with mild glaucoma prioritized the driving task at the expense of missing more and responding slower to the visual symbols. We previously demonstrated that drivers with glaucoma exhibit greater cognitive workload to complete two tasks at the same time.(Gangeddula et al. 2017) Drivers with glaucoma may need to allocate more cognitive resources to the driving task to compensate for their peripheral VF defect. As a result, they respond slower to the VF symbols.

Although the performance-based VF test differs conceptually from static VF tests by adding cognitive demand to the VF task, we still found strong correlations (Spearman rho=0.59) between correct responses in the performancebased VF test and horizontal field of view of the Keystone vision screener. This result indicates that performancebased VF test includes an assessment of physiological VF. In addition, the strong correlation (Spearman rho=-0.53) between correct responses on the performance-based VF test and the UFOV® divided attention in the glaucoma group demonstrates the construct validity of the new test as a measure of functional VF. Considering the small sample size, we also investigated the effect of extreme values. Removal of potential outliers from the analyses still showed moderate strength of correlations. In a larger study that is correctly powered, the influence of outliers should still be treated with extreme caution.

Our sample of drivers with glaucoma did not perform more poorly on the road test compared with control drivers. In fact, most drivers with glaucoma achieved (sub) maximal scores on the road test. This is likely the reason for the lack of significant correlations between scores on the performance-based VF test and on the road test. Future 
research should include a larger group of patients with a broad range of VF defects and driving impairments. The only simulator variable that correlated with on-road driving performance in the glaucoma group was response time on the braking test. Bhorade et al also (2016) found that drivers with glaucoma who performed poorly on the road test responded slower on a braking test compared with those who passed the road test.

In this pilot study, the performance on the VF test indicates that the test has good to excellent test-retest reliability, with ICC values ranging between 0.77 for response time and 0.92 for correct responses. The method of Bland and Altman was used in addition to the ICC values because neither test alone provides sufficient reliability information.(Rankin and Stokes, 1998) The plots confirmed the reliability of the two performance-based VF outcome variables. In addition, the $95 \%$ confidence intervals for the mean difference in performance on the two outcome variables included the value zero, indicating no bias in retesting VF within two weeks after initial testing. However, the width of the $95 \%$ confidence intervals of the ICC values and the Bland Altman graphs showed considerable variability in the individual data. We suggest that in light of the large confidence intervals and our sample size, these findings should be considered very preliminary. Studies with larger sample sizes are needed to test the veracity of our findings.

Simulator technology has made important strides in the last decades. Low-cost driving simulators are now readily available for use in the clinic to screen, assess, or train driving skills for individuals with medical problems.(Classen and Brooks 2014) Whereas driving rehabilitation specialists have very limited access to perimetry tests, they are increasingly using driving simulators to augment their fitness-to-drive assessments.(Classen and Brooks 2014) If concurrent validity is established in studies with larger sample size, this performance-based VF test may replace conventional VF tests such as the Humphrey Visual Field Analyzer, Keystone Vision Screener, or UFOV® test in case these tests are not available.

In conclusion, we have developed a performance-based visual field test to detect visual field impairments while driving in glaucoma. The test has acceptable test-retest reliability and construct validity. Yet, caution with the interpretation is warranted as the study's sample size was small and only included drivers with mild glaucoma. The current results should therefore be considered as exploratory and be confirmed in studies with larger sample size. Future research should also include older individuals with diverse visual field defects and more severe driving impairments to establish concurrent and predictive validity against on-road testing and MVC.

\section{Acknowledgments}

This study was funded by a Public Health Award to H.D. from the Fight for Sight / Prevent Blindness America Foundation and a pilot grant from the Culver Vision Discovery Institute at Augusta University. The funding sources had no involvement in study design, collection, analysis, or interpretation of data. The authors thank Sumner Fishbein, MD, Lane Ulrich, MD, Jason Hughes, MS, Erin Neal, BS, and Dan McGehee, DPT for help with recruitment and administration of tests. We are also grateful for Sanghee Moon, B.S. and Viswa Gangeddula, MS for assistance with data analysis. The authors report no conflict of interest. 


\section{References}

Akinwuntan AE, De Weerdt W, Feys H, Baten G, Arno P, Kiekens C. The validity of a road test after stroke. Arch Phys Med Rehabil. 2005;86:421-426.

Akinwuntan AE, Gantt D, Gibson G, et al. United States version of the Stroke Driver Screening Assessment: A pilot study. Top Stroke Rehabil. 2013;20(1):87-92.

Ball K, Edwards JD, Ross LA. The impact of speed of processing training on cognitive and everyday functions. J Gerontol B Psychol Sci Soc Sci. 2007;62:19-31.

Ball KK, Beard BL, Roenker DL, Miller RL, Griggs DS. Age and visual search: expanding the useful field of view. J Opt Soc Am A. 1988;5(12):2210-2219.

Ball KK, Roenker DL, Bruni JR. Developmental changes in attention and visual search through adulthood. Amsterdam, North Holland: Elsevier; 1990.

Bentley SA, LeBlanc RP, Nicolela MT, Chauhan BC. Validity, reliability, and repeatability of the useful field of view test in persons with normal vision and patients with glaucoma. Invest Ophthalmol Vis Sci. 2012;53(11):6763-6769.

Bhorade AM, Yom VH, Barco P, Wilson B, Gordon M, Carr D. On-road Driving Performance of Patients With Bilateral Moderate and Advanced Glaucoma. Am J Ophthalmol. 2016;166:43-51.

Bland JM, Altman DG. Statistical methods for assessing agreement between two methods of clinical measurement. Lancet. 186:1;307-310.

Carr DB, Schwartzberg JG, Manning L, Sempek J. Physicians guide to assessing and counseling older drivers. American Medical Association; 2010.

Chauhan BC, Garway-Heath DF, Goni FJ, et al. Practical recommendations for measuring rates of visual field change in glaucoma. Br J Ophthalmol. 2008;92(4):569-573.

Cicchetti DV. Guidelines, criteria, and rules of thumb for evaluating normed and standardized assessment instruments in psychology. Psychological Assessment. 1994;6(4):284-290.

Classen S, Brooks J. Driving simulators for occupational therapy screening, assessment, and intervention. Occup Ther Health Care. 2014;28(2):154-162.

Cohen J. A POWER PRIMER. Psychological Bulletin. 1992;112(1):155-159.

Desapriya E, Harjee R, Brubacher J, et al. Vision screening of older drivers for preventing road traffic injuries and fatalities. Cochrane Database Syst Rev. 2014(2):Cd006252.

Dickerson AE, Reistetter T, Davis ES, Monahan M. Evaluating driving as a valued instrumental activity of daily living. Am J Occup Ther. 2011;65(1):64-75.

Edwards JD, Ross LA, Wadley VG, et al. The useful field of view test: Normative data for older adults. Arch Clin Neuropsychol. 2006;21(4):275-286.

Friedman DS, O'Colmain BJ, Munoz B, et al. Prevalence of age-related macular degeneration in the United States. Arch Ophthalmol. 2004;122(4):564-572.

Gangeddula V, Ranchet M, Akinwuntan AE, Bollinger K, Devos H. Effect of cognitive demand on functional visual field performance in senior drivers with glaucoma. Front Aging Neurosci. 2017. 
Gracitelli CP, Tatham AJ, Boer ER, et al. Predicting Risk of Motor Vehicle Collisions in Patients with Glaucoma: A Longitudinal Study. PLoS One. 2015;10(10):e0138288.

Gramer G, Gramer E. Stage of visual field loss and age at diagnosis in 1988 patients with different glaucomas: implications for glaucoma screening and driving ability. Int Ophthalmol. 2018; 38:429-441. Harrabi H, Kergoat MJ, Rousseau J, et al. Age-related eye disease and cognitive function. Invest Ophthalmol Vis Sci. 2015;56(2):1217-1221.

Haymes SA, Leblanc RP, Nicolela MT, Chiasson LA, Chauhan BC. Risk of falls and motor vehicle collisions in glaucoma. Invest Ophthalmol Vis Sci. 2007;48(3):1149-1155.

Haymes SA, LeBlanc RP, Nicolela MT, Chiasson LA, Chauhan BC. Glaucoma and on-road driving performance. Invest Ophthalmol Vis Sci. 2008;49(7):3035-3041.

Jonas JB, Aung T, Bourne RR, Bron AM, Ritch R, Panda-Jonas S. Glaucoma. Lancet. 2017; 390(10108):2183-2193.

Kubler TC, Kasneci E, Rosenstiel W, et al. Driving with Glaucoma: Task Performance and Gaze Movements. Optom Vis Sci. 2015;92(11):1037-1046.

Kunimatsu-Sanuki S, Iwase A, Araie M, et al. An assessment of driving fitness in patients with visual impairment to understand the elevated risk of motor vehicle accidents. BMJ Open. 2015;5(2):e006379.

Kunimatsu-Sanuki S, Iwase A, Araie M, et al. The role of specific visual subfields in collisions with oncoming cars during simulated driving in patients with advanced glaucoma. Br J Ophthalmol. 2017: 101(7):896-901.

Kwon M, Huisingh C, Rhodes LA, McGwin G, Jr., Wood JM, Owsley C. Association between Glaucoma and At-fault Motor Vehicle Collision Involvement among Older Drivers: A Population-based Study. Ophthalmology. 2016;123(1):109-116.

Lee SS, Black AA, Wood JM. Effect of glaucoma on eye movement patterns and laboratory-based hazard detection ability. PLoS One. 2017;12(6):e0178876.

Loughran DS, Seabury SA, Zakaras L. Regulating Older Drivers: Are New Policies Needed? RAND Institute for Civil Justice. 2007.

McGwin G, Jr., Xie A, Mays A, et al. Visual field defects and the risk of motor vehicle collisions among patients with glaucoma. Invest Ophthalmol Vis Sci. 2005;46(12):4437-4441.

Medeiros FA, Weinreb RN, E RB, Rosen PN. Driving simulation as a performance-based test of visual impairment in glaucoma. J Glaucoma. 2012;21(4):221-227.

Nasreddine ZS, Phillips NA, Bedirian V, et al. The Montreal Cognitive Assessment, MoCA: A brief screening tool for mild cognitive impairment. J Am Geriatr Soc. 2005;53(4):695-699.

National Highway Traffic Safety Administration. Traffic Safety Facts - Older Population - 2015 Data. US Department of Transportation. 2017.

Nouri FM, Lincoln NB. Predicting driving performance after stroke. BMJ Open. 1993;307(6902):482-483. Owsley C, McGwin Jr G, Ball K. Vision impairment, eye disease, and injurious motor vehicle crashes in the elderly. Ophthalmic Epidemiology. 1998;5(2):101-113. 
Prado Vega R, van Leeuwen PM, Rendon Velez E, Lemij HG, de Winter JC. Obstacle avoidance, visual detection performance, and eye-scanning behavior of glaucoma patients in a driving simulator: a preliminary study. PLoS One. 2013;8(10):e77294.

Rankin G, Stokes M. Reliability of assessment tools in rehabilitation: an illustration of appropriate statistical analysis. Clinical Rehabilitation. 1998:12:187-199.

Reitan RM. Trail Making Test Manual for Administration and Scoring. Tucson, AZ: Reitan Neuropsychology Laboratory; 1986.

Steinkuller P. Legal Vision Requirements for Drivers in the United States. AMA J Ethics. 2010;12(12):938940.

Szlyk JP, Taglia DP, Paliga J, Edward DP, Wilensky JT. Driving performance in patients with mild to moderate glaucomatous clinical vision changes. J Rehabil Res Dev. 2002;39(4):467-481.

Szlyk JP, Mahler CL, Seiple W, Edward DP, Wilensky JT. Driving performance of glaucoma patients correlates with peripheral visual field loss. J Glaucoma. 2005;14(2):145-150.

Tatham AJ, Boer ER, Gracitelli CP, Rosen PN, Medeiros FA. Relationship Between Motor Vehicle Collisions and Results of Perimetry, Useful Field of View, and Driving Simulation in Drivers With Glaucoma. Transl Vis Sci Technol. 2015;4(3):5.

Wilkie RM, Mole CD. Looking to the future of visual assessment using driving simulation. Scandinavian J Optomet Vis Sci. 2017;10(1):8-17.

Wood JM, Black AA. Ocular disease and driving. Clin Exp Optom. 2016;99(5):395-401.

Wood JM, Black AA, Mallon K, Thomas R, Owsley C. Glaucoma and Driving: On-Road Driving Characteristics. PLoS One. 2016;11(7):e0158318.

Yuki K, Asaoka R, Awano-Tanabe S, et al. Predicting Future Self-Reported Motor Vehicle Collisions in Subjects with Primary Open-Angle Glaucoma Using the Penalized Support Vector Machine Method.

Transl Vis Sci Technol. 2017;6(3):14. 
Figure 1. Screenshot of driving scene (100 degrees horizontal field of view, 20 degrees vertical field of view) with example target symbol in the central visual field

Figure 2. Bland-Altman plot for test-retest reliability performance-based VF test response time (A) and correct responses (B). The mean response time (A) and mean correct responses (B) of the test and re-test are plotted against the difference in scores between test and retest. The solid line represents the mean of the differences. The dashed lines define the limits of agreement (mean of the difference $\pm 2 \mathrm{SD}$ ).

Supplementary Figure 1: Low-fidelity driving simulator (@STISIM drive)

Supplementary Figure 2. All 114 target symbols displayed on the screen (100 degrees horizontal field of view, 20 degrees vertical field of view) 